\title{
Interplay between lattice, orbital, and magnetic degrees of freedom in the chain-polymer $\mathrm{Cu}(\mathrm{II})$ breathing crystals
}

\author{
S. V. Streltsov, ${ }^{1,2, *}$ M. V. Petrova, ${ }^{3}$ V. A. Morozov, ${ }^{3}$ G. V. Romanenko, ${ }^{3}$ V. I. Anisimov, ${ }^{1,2}$ and N. N. Lukzen ${ }^{3}$ \\ ${ }^{1}$ Institute of Metal Physics, S. Kovalevskoy St. 18, 620990 Ekaterinburg, Russia \\ ${ }^{2}$ Ural Federal University, Mira St. 19, 620002 Ekaterinburg, Russia \\ ${ }^{3}$ International Tomography Center SB RAS, Institutskaya Str. 3a, Novosibirsk, Russia
}

(Received 9 August 2012; revised manuscript received 27 November 2012; published 31 January 2013)

\begin{abstract}
The chain-polymer $\mathrm{Cu}(\mathrm{II})$ "breathing crystals" $\mathrm{C}_{21} \mathrm{H}_{19} \mathrm{CuF}_{12} \mathrm{~N}_{4} \mathrm{O}_{6}$ were studied using the x-ray diffraction and $a b$ initio band structure calculations. We show that the crystal structure modification at $T=146 \mathrm{~K}$, associated with the spin crossover transition, induces the changes of the orbital order in half of the $\mathrm{Cu}$ sites. This in turn results in the switch of the magnetic interaction sign in accordance with the Goodenough-Kanamori-Andersen theory of the coupling between the orbital and spin degrees of freedom.
\end{abstract}

DOI: 10.1103/PhysRevB.87.024425

PACS number(s): 75.30.Wx, 61.66.Hq, 31.15.A-

\section{INTRODUCTION}

The conventional phenomenon of spin crossover (SCO) is a well-known change of the spin state observed in some octahedral coordinated transition metal complexes. ${ }^{1}$ There exist thermally, doping, photo-, and pressure induced SCO transitions. ${ }^{1-4}$ In the classical case of Fe(II) complexes with $d^{6}$ electron configuration, the thermal SCO involves a transition from the low spin state $\left(S=0, t_{2 g}^{6}\right)$ to the high spin state $\left(S=2, t_{2 g}^{4} e_{g}^{2}\right)^{5,6}$ at elevating temperature. The SCO compounds represent bright examples of a bistability in the molecular crystals ${ }^{7}$ and are promising candidates for multifunctional materials ${ }^{8}$ with potential applications to the memory devices, the optical, temperature, and pressure sensors, etc. ${ }^{9}$

Recently rather different SCO transitions were found in the chain-polymer compounds $\mathrm{Cu}$ (II) with nitroxides. ${ }^{10}$ These compounds contain chains of the exchange clusters with two or three spins. The exchange clusters contain $\mathrm{Cu}^{2+}$, ligands, and some organic radicals. For the essential structural changes in the polyhedral surrounding $\mathrm{Cu}$ ions under the $\mathrm{SCO}$ transitions, these compounds were called "breathing crystals."11 The main feature of the breathing crystals compounds is their ability to pass through reversible thermal induced structural transformations (often similar to phase transitions) accompanied by changes of magnetic susceptibility and optical properties. ${ }^{12}$ Note that one should not confuse the breathing crystal with the well known dynamical "breathing mode"-a specific collective excitation of confined systems of quantum ${ }^{13}$ or classical ${ }^{14}$ particles.

The classical SCO transition, associated with the change of spin state of a single ion, is impossible for isolated $d^{9}$ centers of $\mathrm{Cu}(\mathrm{II})\left(S=1 / 2, t_{2 g}^{6} e_{g}^{3}\right)$. Thus, the reason for unusual spin transitions inherent in the $\mathrm{Cu}(\mathrm{II})$ complexes with nitroxides possibly arise from the change of the total electron spin of a whole exchange cluster.

Magnetic measurements show that the temperature induced SCO transition in one of the $\mathrm{Cu}$ (II) breathing crystals, characterized by the chemical formula $\mathrm{C}_{21} \mathrm{H}_{19} \mathrm{CuF}_{12} \mathrm{~N}_{4} \mathrm{O}_{6}$, is accompanied by the lost of half of the local spins. ${ }^{11}$ This fact can be explained by the formation of spin singlets $(S=0)$ in half of the exchange clusters, but the reason for this is unknown.

The aim of our paper is to provide a microscopic description of the changes in the magnetic properties of
$\mathrm{C}_{21} \mathrm{H}_{19} \mathrm{CuF}_{12} \mathrm{~N}_{4} \mathrm{O}_{6}$, often abbreviated as $\mathrm{Cu}(\mathrm{hfac})_{2} \mathrm{~L}^{M e}$ in the chemical literature. With the use of the density functional theory (DFT) we found that there is an interplay between the magnetic properties, orbital structure, and lattice distortions in the breathing crystals. These correlations between different degrees of freedom result in the SCO transition at $146 \mathrm{~K}$ in the compound under consideration.

\section{CRYSTAL STRUCTURE}

The crystal structure of $\mathrm{C}_{21} \mathrm{H}_{19} \mathrm{CuF}_{12} \mathrm{~N}_{4} \mathrm{O}_{6}$ was solved from the $\mathrm{x}$-ray single crystals diffraction data. The data were collected using a SMARTAPEXCCD (Bruker AXS) automated diffractometer with a helix (Oxford Cryosystems) open-flow helium cooler using the standard procedure (Mo $K \alpha$ radiation). The structures were solved by direct methods and refined by the full-matrix least-squares procedure anisotropically for nonhydrogen atoms. The $\mathrm{H}$ atoms were partially located in different electron density syntheses, and the others were calculated geometrically and included in the refinement as riding groups. All calculations were fulfilled with the SHELXTL 6.14 program package.

Crystal data for the compound $\mathrm{Cu}(\mathrm{hfac})_{2} \mathrm{~L}^{\mathrm{Me}}$ are the following: $\mathrm{C}_{21} \mathrm{H}_{19} \mathrm{CuF}_{12} \mathrm{~N}_{4} \mathrm{O}_{6}, \quad F W=714.94, \quad T=$ $240 / 110 \mathrm{~K}$, triclinic crystals, space group $P-1, a=$ 12.1987(9)/11.9560(9) $\AA, b=15.5950(11) / 15.0506(12) \AA$, $c=15.8716(11) / 15.8657(12) \AA, \alpha=84.459(2) / 81.306(1)^{\circ}$, $\beta=74.132(2) / 76.943(2)^{\circ}, \gamma=87.315(1) / 85.502(1)^{\circ}, V=$ $2890.1(4) / 2746.3(4) \AA^{3}, Z=4, D_{\text {calc }}=1.643 / 1.729 \mathrm{~g} / \mathrm{cm}^{3}$, $\mu=0.875 / 0.921 \mathrm{~mm}^{1}, 12570 / 11868$ measured reflections $\left(\theta_{\max }=23.35 / 23.30^{\circ}\right), 8291 / 7907$ unique reflections $\left(R_{\text {int }}=\right.$ $0.0293 / 0.0230), 6043 / 6521$ reflections with $I>2 \sigma_{I}$, $793 / 794$ refined parameters; $G O O F=1.061 / 1.103, R 1=$ $0.0598 / 0.0481, w R 2=0.1500 / 0.1307\left(I>2 \sigma_{I}\right)$. The list of the atomic positions for $T=240 \mathrm{~K}$ and $T=110 \mathrm{~K}$ is given in the Supplemental Material. ${ }^{15}$

The crystal structure of $\mathrm{C}_{21} \mathrm{H}_{19} \mathrm{CuF}_{12} \mathrm{~N}_{4} \mathrm{O}_{6}$ consists of the polymer chains running along $b$ direction (Fig. 1) with a "head-to-tail" motif containing the exchange clusters of type $\mathrm{N}-\mathrm{O}-\mathrm{Cu}^{2+}$. An isolated chain of the exchange clusters is presented in Fig. 2 with the $\mathrm{F}$ and $\mathrm{H}$ atoms being omitted for simplicity. 


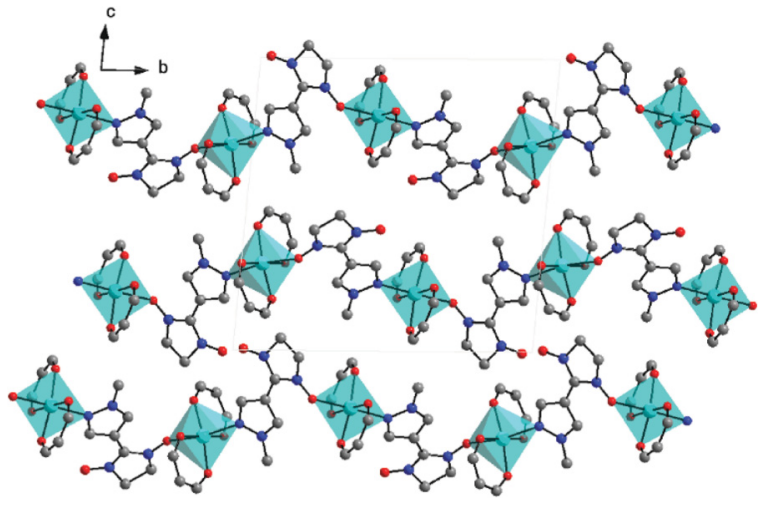

FIG. 1. (Color online) The crystal structure of high-temperature triclinic phase of the breathing crystal compound $\mathrm{C}_{21} \mathrm{H}_{19} \mathrm{CuF}_{12} \mathrm{~N}_{4} \mathrm{O}_{6}$. Turquoise balls are the $\mathrm{Cu}$ ions, red and blue balls are the $\mathrm{O}$ and $\mathrm{N}$ atoms. Gray balls are the $\mathrm{C}$ atoms. The fluorine atoms and the hydrogen atoms are omitted for clarity of the figure. Coordination units $\mathrm{CuO}_{5} \mathrm{~N}$ are marked with turquoise octahedra.

At high-temperature (HT) phase the $\mathrm{CuO}_{5} \mathrm{~N}$ units are the elongated octahedra with a $\mathrm{Cu}-\mathrm{O}$ axial distance of $\sim 2.5 \AA$ and $\mathrm{Cu}-\mathrm{N}$ distance of $\sim 2.3 \AA$. Equatorial distances $\mathrm{Cu}-\mathrm{O}$ are about $1.95 \AA$. With decrease of the temperature the compound undergoes the SCO transition in the vicinity of $150 \mathrm{~K}$, which is accompanied by substantial structural changes. As a result of the transition within half of the $\mathrm{CuO}_{5} \mathrm{~N}$ octahedra, the elongation direction changes. In the octahedra surrounding
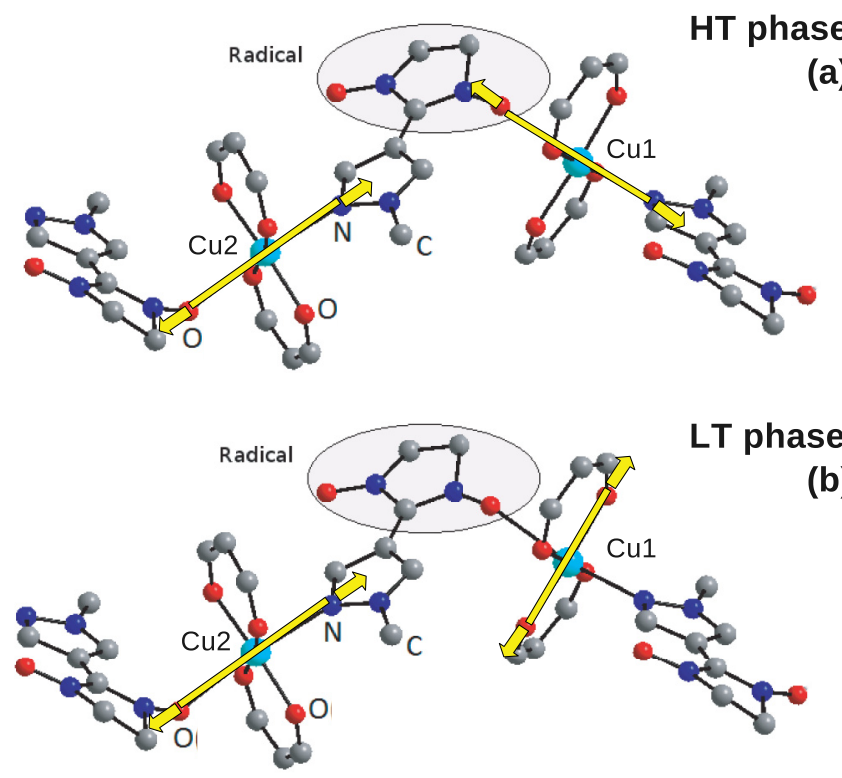

FIG. 2. (Color online) The structure of isolated polymer chain of the breathing crystal compound $\mathrm{C}_{21} \mathrm{H}_{19} \mathrm{CuF}_{12} \mathrm{~N}_{4} \mathrm{O}_{6}$. The color coding is the same as in Fig. 1 The fluorine atoms and the hydrogen atoms are omitted for clarity of the figure. Methyl ligand characterizing this compound is marked by $\mathrm{C}$. In other compounds of the breathing crystals family the ligand may be propyl or butyl type. A fragment of nitroxide radical with nonzero spin densities on atoms is marked with the oval. The direction of the $\mathrm{CuO}_{5} \mathrm{~N}$ octahedra elongation are shown by the yellow bars and arrows.
TABLE I. Bond distances of $\mathrm{Cu} 2 \mathrm{O}_{5} \mathrm{~N}$ octahedra in $\AA$. The first four rows correspond to distances in the equatorial plane, i.e., perpendicular to the chain direction, while the last two to the axial direction (along the chain). The space group is the same $P-1$ for both phases.

\begin{tabular}{lccccc}
\hline \hline & HT phase & LT phase & & HT phase & LT phase \\
Bond & $T=240 \mathrm{~K}$ & $T=110 \mathrm{~K}$ & Bond & $T=240 \mathrm{~K}$ & $T=110 \mathrm{~K}$ \\
\hline $\mathrm{Cu} 1-\mathrm{O}$ & 1.95 & 1.97 & $\mathrm{Cu} 2-\mathrm{O}$ & 1.93 & 1.85 \\
$\mathrm{Cu} 1-\mathrm{O}$ & 1.95 & 2.16 & $\mathrm{Cu} 2-\mathrm{O}$ & 1.95 & 1.85 \\
$\mathrm{Cu} 1-\mathrm{O}$ & 1.96 & 1.98 & $\mathrm{Cu} 2-\mathrm{O}$ & 1.96 & 1.97 \\
$\mathrm{Cu} 1-\mathrm{O}$ & 1.97 & 2.23 & $\mathrm{Cu} 2-\mathrm{O}$ & 1.97 & 1.97 \\
$\mathrm{Cu} 1-\mathrm{O}$ & 2.49 & 1.99 & $\mathrm{Cu} 2-\mathrm{O}$ & 2.49 & 2.42 \\
$\mathrm{Cu} 1-\mathrm{N}$ & 2.30 & 2.02 & $\mathrm{Cu} 2-\mathrm{N}$ & 2.32 & 2.32 \\
\hline \hline
\end{tabular}

the $\mathrm{Cu} 1$ ion two equatorial bonds with oxygen turn out to be the longest, while in the $\mathrm{Cu}_{2} \mathrm{O}_{5} \mathrm{~N}$ octahedra the elongation direction still coincides with the $\mathrm{O}-\mathrm{Cu}-\mathrm{N}$ bond, like in the HT phase. Corresponding bond lengths can be found in Table I for the HT $(240 \mathrm{~K})$ and LT $(110 \mathrm{~K})$ phases.

\section{PREVIOUS CALCULATIONS OF THE BREATHING CRYSTALS}

The first attempt to understand the nature of the spin exchange in the clusters containing the $\mathrm{Cu}$ atoms and a stable nitroxyl radical was performed rather long ago by Musin et al. ${ }^{16}$ The authors provided a detail quantumchemical analysis of the possible mechanisms of the exchange interaction in the magnetic fragments $\mathrm{Cu}(\mathrm{II}) \cdots \mathrm{O}-\mathrm{N}<($ or $>\dot{\mathrm{N}}$ $\mathrm{O} \cdots \dot{\mathrm{Cu}}(\mathrm{III}) \cdots \mathrm{O}-\dot{\mathrm{N}}<$ ) of bischelating complexes of $\mathrm{Cu}(\mathrm{II})$ with nitroxyl radicals. The drawback of this and some other ${ }^{17-20}$ treatments was in the consideration of an isolated fragment rather than the crystal as a whole.

Till now the only consistent calculation of the electronic and magnetic properties of the breathing crystals was performed in Ref. 21, where the spin densities and the magnetic moments of a heterospin compound based $\mathrm{Cu}(\mathrm{II})$ hexafluoroacetylacetonate $\left[\mathrm{Cu}(\mathrm{hfac})_{2}\right.$; hfac $\left.=\mathrm{CF}_{3}-\mathrm{C}(\mathrm{O})-\mathrm{CH}-\mathrm{C}(\mathrm{O})-\mathrm{CF}_{3}\right]$ in combination with a stable nitronyl nitroxide radical were calculated. This system is similar to the one of interest in the present paper, the difference is in another substitute in position 1 of the pyrazol ring - the ethyl radical instead of the methyl one in our system. This difference results in another organization of the polymer chain: In the case considered in Ref. 21 the chain of the exchange clusters has "head-to-head" coupling of the ligands to the magnetic $\mathrm{Cu}$ atoms embedded in (hfac) $)_{2}$ blocks, while in the present case the chain motif is "head-to-tail."

Thus, in Ref. 21 the corresponding chain contains a "three spin-isolated spin" structure, while in the case considered in the present paper the chain is composed of two spin clusters. This results in different magnetic properties. It should also be mentioned that in Ref. 21 only a high-temperature phase of the crystal was calculated. At the same time, both hightemperature and especially low-temperature phase represent a great interest for study of phase transitions. 


\section{CALCULATION DETAILS}

The pseudopotential PW-SCF code was used for the band structure calculations. ${ }^{22}$ We utilized ultrasoft pseudopotentials with nonlinear core correction (for better description of the magnetic interactions) with the Perdew-Burke-Ernzerhof (PBE) version of the exchange-correlation potential. ${ }^{23}$ The charge density and kinetic energy cutoffs equal 35 and $180 \mathrm{Ry}$, respectively. The integration in the $k$ space in the course of the self-consistency was performed over the mesh of four $k$ points in the Brillouin zone using Gaussian smearing of $13.6 \mathrm{meV}$. The density of states was calculated with the smearing of $40.8 \mathrm{meV}$.

The correlations on $\mathrm{Cu}$ sites were taken into account within the frameworks of the GGA + U approximation (generalized gradient approximation with account of on-site Coulomb repulsion). ${ }^{24}$ The intra-atomic exchange interaction $J_{H}$ and on-site Coulomb repulsion parameters for $\mathrm{Cu}^{2+}$ ions were chosen to be 0.9 and $7.0 \mathrm{eV}$, respectively. ${ }^{25,26}$

The calculations were performed for the experimentally measured crystal structure, presented in Ref. 15. However, in order to decrease the number of atoms in the unit cell (from 252 to 126 ) we used $P 1$ instead of the $P-1$ space group. The inversion center in the $P-1$ space group produces additional chains, so that in reducing the crystal symmetry we neglect the interchain interactions. This seems to be a reasonable approximation since the main changes in the crystal structures at the SCO transition occur within a chain.

\section{CAlCulation ReSUlts}

\section{A. High-temperature phase}

We start with the calculations of the HT phase. The compound under consideration is experimentally known to be paramagnetic. ${ }^{11}$ However, in any band structure calculation the translational symmetry is assumed, so that one may calculate the ferromagnetic (FM), when both $\mathrm{Cu}$ ions in the unit cell (u.c.) have the same spin direction, antiferromagnetic, with the opposite spins on the neighboring $\mathrm{Cu}$ atoms, or nonmagnetic, when up and down spins on all ions are equally populated. It is clear that the nonmagnetic configuration is the worst approximation since there must be local magnetic moments in the paramagnetic insulator. In the following we will investigate the FM and AFM solutions, which actually provide very similar results since magnetic ions $(\mathrm{Cu})$ are quite far away from each other.

Indeed, the absolute values of the magnetic moments on two $\mathrm{Cu}$ ions were found to be 0.52 and $0.55 \mu_{B}$, the same for the FM and AFM solutions (see Table II). They differ from the $1 \mu_{B}$ expected for the isolated $\mathrm{Cu}^{2+}$ ions due to a strong hybridization with ligands and formation of a molecular orbital on which a single hole in the $3 d$ shell of the $\mathrm{Cu}^{2+}$ ion is localized. This molecular orbital (or the Wannier orbital) has the contributions of the $\mathrm{Cu} d$ and ligand $p$ orbitals, so that the spin moment on the whole molecular orbital should be $1 \mu_{B}$, but the part of the spin density projected on the $\mathrm{Cu}$ $3 d$ states provides only a part of it. This is clearly seen from the density of states plot presented in Fig. 3. The peak at $\sim 0.1 \mathrm{eV}$ corresponding to a single hole in the $\mathrm{Cu} 3 d$ shell shows significant contribution coming from the $\mathrm{O} 2 p$ states.
TABLE II. The results of the GGA + $\mathrm{U}$ calculation for two different crystal structures corresponding to the temperatures $T=$ $110 \mathrm{~K}$ (LT) and $T=240 \mathrm{~K}$ (HT) and to the FM and AFM types of magnetic order of the $\mathrm{Cu}$ spins. All the values are in $\mu_{B}$ units. The total and absolute (Abs) magnetization are per unit cell.

\begin{tabular}{lcrcr}
\hline \hline & $\begin{array}{c}\text { HT phase } \\
\text { FM }\end{array}$ & $\begin{array}{c}\text { HT phase } \\
\text { AFM }\end{array}$ & $\begin{array}{c}\text { LT phase } \\
\text { FM }\end{array}$ & $\begin{array}{c}\text { LT phase } \\
\text { AFM }\end{array}$ \\
\hline Total magn. & 3.92 & 0.06 & 2.00 & 2.00 \\
Abs magn. & 4.40 & 4.44 & 4.07 & 4.07 \\
Moment Cu1 & 0.52 & -0.52 & 0.45 & -0.45 \\
Moment Cu2 & 0.55 & 0.55 & 0.55 & 0.55 \\
\hline \hline
\end{tabular}

Another illustration of the considerable mixing between $\mathrm{Cu}$ $3 d$ and $\mathrm{O} 2 p$ states can be found in Figs. 5 and 7, where the spin density plot for the LT phase is presented.

It is useful to proceed with the analysis of the total magnetization per unit cell defined as $m_{\text {tot }}=\sum_{i} m_{i}$, where $m_{i}=g \mu_{B} s_{i}$ is the magnetization on the $i$ th atom, and $s_{i}$ is spin moment. The total magnetization equals $3.92 \mu_{B}$ in the FM and $0.06 \mu_{B}$ in the AFM configuration. Since there are two radical- $\mathrm{Cu}$ pairs in the unit cell, it means that at least in the FM configuration the spins on the radicals are parallel to the spin moment of the molecular orbital on the neighboring (to this radical) $\mathrm{Cu}$ ion. The spin density projection shows that the largest moment on $p$ elements $(\mathrm{O}, \mathrm{C}, \mathrm{F}$, and $\mathrm{N})$ are indeed parallel to the moments of $\mathrm{Cu}$. The values of the largest moments $\left(>0.1 \mu_{B}\right)$ in the case of the FM order are the following: O10 $\left(0.24 \mu_{B}\right)$; O11 $\left(0.30 \mu_{B}\right)$; O12 $\left(0.31 \mu_{B}\right)$; N1, N2, N5 $\left(0.22 \mu_{B}\right)$; N6 $\left(0.22 \mu_{B}\right)$.

In other words, the radical-Cu pair is in the triplet ground state ( $S=1$, where $S$ is the spin of the pair). The deviation from the $4 \mu_{B}$ for the FM and from $0 \mu_{B}$ for the AFM solutions is attributed to the sparse mesh in the $k$ space, used to integrate energy bands in such a large unit cell, consisting of 126 atoms.

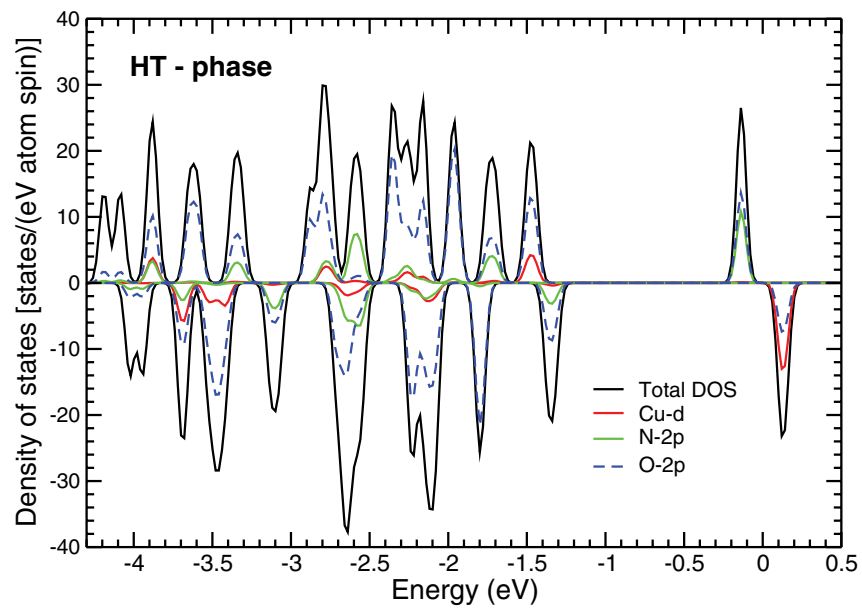

FIG. 3. (Color online) Results of the GGA + U calculation for the high-temperature phase, where the spins on different $\mathrm{Cu}$ ions are ferromagnetically ordered. Positive (negative) values correspond to the spin up (down). The Gaussian smearing $0.04 \mathrm{eV}$ was used. The Fermi energy is set to zero. 
In principle, one may explain the ferromagnetic coupling (parallel spin arrangement) of the spins on the $\mathrm{Cu}$ ions and radicals in two ways. First of all one may argue that the local spin on $\mathrm{Cu}$ simply magnetizes all the surrounding ions. Microscopically this means that due to a strong hybridization the Zeeman (spin) splitting in the $\mathrm{Cu} 3 d$ shell spreads out on the $s$ and $p$ shells of the neighboring ligands. However, this picture is too simplified and does not take into account the details of the electronic structure of the compound under consideration and is unable to explain the antiferromagnetic coupling between $\mathrm{Cu}$ and the radical which is observed at low temperatures and will be discussed latter. Therefore below we present the model which explains in detail how the magnetic coupling with the radicals is related to the local lattice distortions and the orbital structure of the $\mathrm{Cu} 3 d$ shell.

According to the crystal structure analysis presented above in Sec. II, the distortions of both $\mathrm{CuO}_{5} \mathrm{~N}$ octahedra (which belong to the same unit cell) are quite similar. Since the $\mathrm{Cu}^{2+}$ ion is Jahn-Teller active, both octahedra are strongly distorted. They are elongated in the direction of the $\mathrm{O}-\mathrm{Cu}-\mathrm{N}$ bond. The average $\mathrm{Cu}-\mathrm{O}$ distance in the equatorial plane is $\sim 1.95 \AA$, while the bond lengths with the apical ligands are $\sim 2.30$ and $2.49 \AA$ for the $\mathrm{Cu}-\mathrm{O}$ and $\mathrm{Cu}-\mathrm{N}$ bonds, respectively.

Such a distortion of the local surrounding of the $\mathrm{Cu}^{2+}$ leads to a certain splitting in the $e_{g}$ shell of these ions: The orbital of the $x^{2}-y^{2}$ symmetry turns out to be higher in energy than $3 z^{2}-r^{2}$, as it is shown in Fig. 4. As a result the hole localizes on this $x^{2}-y^{2}$ molecular orbital, which lies in the plane orthogonal to the bond with the radical. By symmetry this orbital may hybridize only with the $\mathrm{O} 2 p$ states, not with the $\mathrm{N} 2 p$ orbitals. This is clearly seen in Fig. 3, where the peak corresponding to the hole states at $\sim 0.1 \mathrm{eV}$ does not have the contribution coming from the $\mathrm{N} 2 \mathrm{p}$ states.

Thus the overlap between magneto-active orbital centered on the $\mathrm{Cu}^{2+}$ ion and the molecular orbital bearing the local spin on the radical is negligible. The only possible magnetic coupling between $\mathrm{Cu}$ and the radical is via orbital of the $3 z^{2}-r^{2}$ symmetry. But this interaction between the completely filled $3 z^{2}-r^{2}$ orbital and the partially filled radical molecular orbital must be ferromagnetic according to the famous Goodenough-Kanamori-Andersen (GKA) rules. ${ }^{27}$ This is exactly what we observe in the calculation.

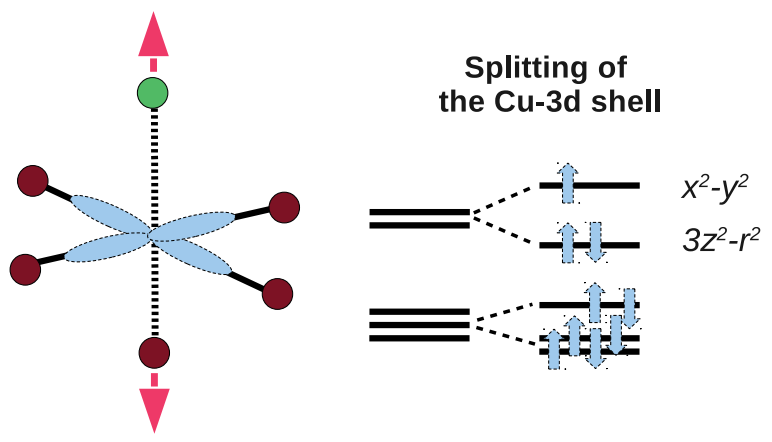

FIG. 4. (Color online) The sketch of the local distortion of $\mathrm{CuO}_{5} \mathrm{~N}$ octahedra (oxygen and nitrogen atoms are brown and green balls, respectively) in the high-temperature phase. The direction of the distortion is shown by the red arrows. The elongation of the octahedra leads to the stabilization of a hole on the $x^{2}-y^{2}$ orbital.

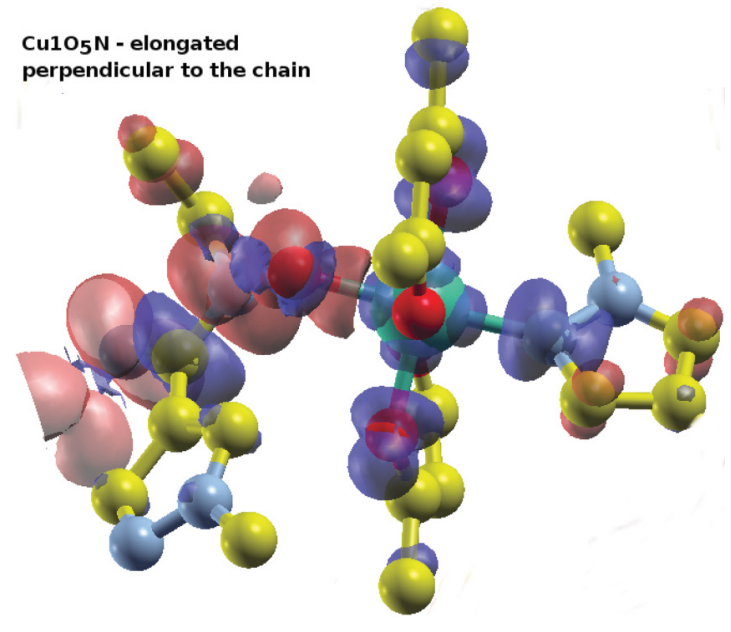

FIG. 5. (Color online) The spin density $\left[\rho^{\uparrow}(\vec{r})-\rho^{\downarrow}(\vec{r})\right]$ in the vicinity of the $\mathrm{Cu} 1$ ion obtained in the GGA + $\mathrm{U}$ calculation for $\mathrm{LT}$ phase. The $\mathrm{Cu}, \mathrm{N}, \mathrm{O}$, and $\mathrm{C}$ ions are shown as light green, light blue, red, and yellow balls, correspondingly. The parts of the spin density, which have different signs, are painted by different colors: brown and violet. Thus, one may easily seen that the spins on the radical and on the $\mathrm{Cu} 1$ are antiparallel. The plot is for the FM order of $\mathrm{Cu}$ spins, but the AFM order gives qualitatively the same.

\section{B. Low-temperature phase}

The situation in the low-temperature (LT) phase is more complicated, mainly due to the change in the direction of the elongation in half of the $\mathrm{CuO}_{5} \mathrm{~N}$ octahedra. In the LT phase the octahedra surrounding the $\mathrm{Cu} 1$ ions turn out to be elongated not in the direction of the chain, but perpendicular to it. This is schematically shown in Fig. 2(b). The change of the elongation direction results in the rotation of the single magneto-active orbital on the $\mathrm{Cu} 1$ ion. The symmetry of this orbital must be the same, $x^{2}-y^{2}$ (here we used the notations of the local coordinate system, where $z$ axis corresponds to elongation direction), but two lobes of the orbital must point to the radical.

In Fig. 5 the spin density (difference between the charge densities for two spin projections) around the $\mathrm{Cu} 1$ ion obtained in the GGA + U calculation is shown. In the case of the $\mathrm{Cu}^{2+}$ $\left(d^{9}\right)$ this corresponds to the spatial distribution of the single unoccupied orbital. One may see that, as it was described in detail in the previous section, the single hole is actually stabilized not on the atomic but on the molecular (Wannier) orbital, which has significant contribution on the neighboring ligands. The symmetry of the orbital is $x^{2}-y^{2}$, but it is pointed exactly at the spin density centered on the radical (left part of Fig. 5). The strong overlap between the half-filled orbitals centered on the $\mathrm{Cu} 1$ ion and on the radical results in the strong antiferromagnetic coupling $J \sim 2 t^{2} / U$ according to the GKA rules. ${ }^{27}$ Here $t$ is the hopping integral and $U$ is the on-site Coulomb repulsion parameter. The fact that this superexchange interaction in the real calculation does lead to the antiferromagetic order of the spins on the radical and $\mathrm{Cu} 1$ is clearly seen from Fig. 5. The signs of the spin density on these two objects are indeed different.

The presence of the molecular orbital centered on the radical with the spin antiparallel to the spin on one of the $\mathrm{Cu}$ ions is 


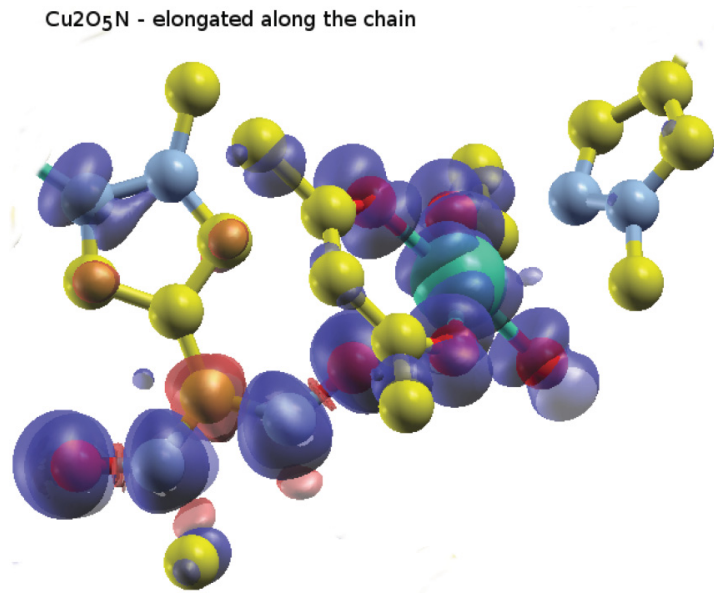

FIG. 6. (Color online) The spin density $\left[\rho^{\uparrow}(\vec{r})-\rho^{\downarrow}(\vec{r})\right]$ in the vicinity of the $\mathrm{Cu} 2$ ion obtained in the GGA + U calculation for LT phase. The $\mathrm{Cu}, \mathrm{N}, \mathrm{O}$, and $\mathrm{C}$ ions are shown as light green, light blue, red, and yellow balls, correspondingly. The parts of the spin density, which have different signs, are painted by different colors: brown and violet. Thus, the spins on the radical and on the $\mathrm{Cu} 2$ are parallel. The plot is for the FM order of $\mathrm{Cu}$ spins, but the AFM order gives qualitatively the same.

also seen in Fig. 7. In contrast to the HT phase there are two peaks above the Fermi level. One of them has contributions coming from the $\mathrm{Cu} 3 d, \mathrm{~N} 2 p$, and $\mathrm{O} 2 p$ states, while another only from the $\mathrm{N} 2 p$ and $\mathrm{O} 2 p$ states. The last one has the spin projection opposite to the spins on the $\mathrm{Cu}$ ions.

The spin density centered on the $\mathrm{Cu} 2$ ion is presented in Fig. 6. One may see that it is concentrated in the plane perpendicular to the charge density of the radical (so called antiferro-orbital ordering). This leads to the FM coupling between $\mathrm{Cu} 2$ and the neighboring radical, as in the HT phase according to the GKA rules. Indeed the sign of the spin density is the same on the radical as on the $\mathrm{Cu} 2$ ion.

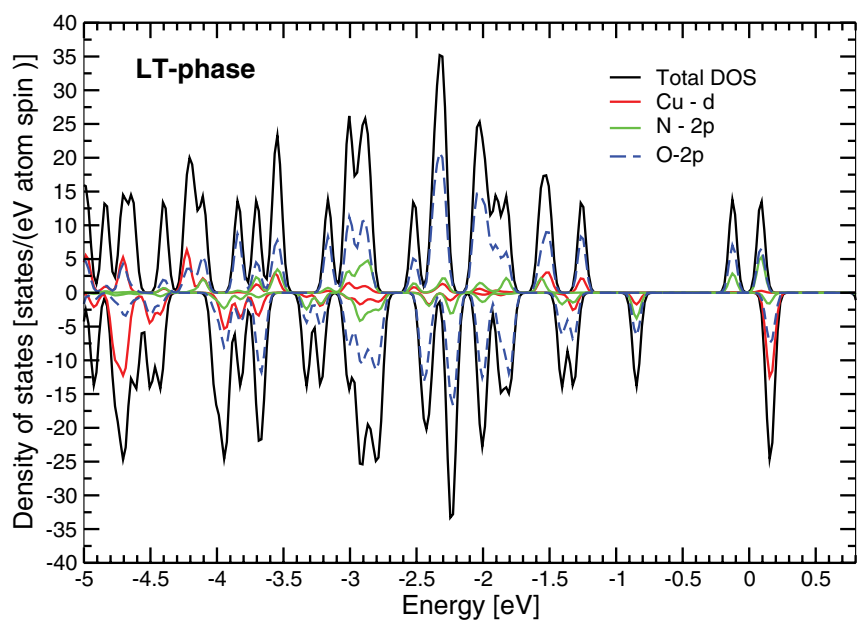

FIG. 7. (Color online) Results of the GGA + U calculation for the low-temperature phase, where the spins on different $\mathrm{Cu}$ ions are ferromagnetically ordered. Positive (negative) values correspond to the spin up (down). The Gaussian smearing $0.04 \mathrm{eV}$ was used. The Fermi energy is set to zero.
The fact that there must be a different magnetic coupling in two $\mathrm{Cu}$-radical pairs in the LT phase according to the charge density analysis is also seen from the values of the total and absolute magnetic moments per unit cell. The total magnetic moment per unit cell is the same, $2.0 \mu_{B}$ in both FM and AFM configurations, which shows that there is one pair of electrons on the $\mathrm{Cu}^{2+}$ ion and the radical with the parallel spin direction and another one with the antiparallel spins. The fact that the absolute magnetization defined as $m_{\text {abs }}=\sum_{i}\left|m_{i}\right|$ is equal to $4.07 \mu_{B}$ additionally supports this interpretation.

The absolute values of magnetic moments were found to be the same in the FM and AFM solutions and equal to 0.45 and $0.55 \mu_{B}$ for the $\mathrm{Cu} 1$ and $\mathrm{Cu} 2$ ions, respectively [a difference in the values of magnetic moments as compared to the high-temperature phase can be connected with a slightly different hybridization with ligands, due to different $\mathrm{Cu}-\mathrm{O}(\mathrm{N})$ distances].

The strong antiferromagnetic coupling results in the spin singlet state $(S=0)$ formation in the $\mathrm{Cu} 1$ exchange clusters for which the $\mathrm{CuO}_{5} \mathrm{~N}$ octahedra are elongated perpendicular to chain direction. This is exactly what is observed experimentally - the lost of the half of localized spins in the low-temperature phase. ${ }^{11}$

\section{CONCLUSIONS}

We present a microscopic theory which describes anomalous changes of the magnetic properties of $\mathrm{C}_{21} \mathrm{H}_{19} \mathrm{CuF}_{12} \mathrm{~N}_{4} \mathrm{O}_{6}$ breathing crystal through the coupling between the spins and another degrees of freedom such as orbital and lattice.

With the use of the $a b$ initio band structure calculations we show that the change of the crystal structure of $\mathrm{C}_{21} \mathrm{H}_{19} \mathrm{CuF}_{12} \mathrm{~N}_{4} \mathrm{O}_{6}$ with decrease of the temperature results in the rotation of the half-filled orbital of one of the $\mathrm{Cu}^{2+}$ ions in the unit cell. The $x^{2}-y^{2}$ orbital lies in the plane orthogonal to the $\mathrm{O}-\mathrm{Cu}-\mathrm{N}$ bond and provides ferromagnetic coupling at high temperatures. With a decrease of temperature the distortions of the half of the $\mathrm{CuO}_{5} \mathrm{~N}$ octahedra are changed, so that two lobes of the $x^{2}-y^{2}$ orbital on the $\mathrm{Cu} 1$ sites turn out to be directed along the $\mathrm{O}-\mathrm{Cu}-\mathrm{N}$ bond. This leads to a strong antiferromagnetic coupling and stabilization of the spin singlet $(S=0)$ state in half of the exchange clusters at lower temperatures. As a result, only half of the local spins of the compound turns out to be unpaired and the value of observed effective magnetic moment drastically drops. ${ }^{11,20}$

\section{ACKNOWLEDGMENTS}

The authors are thankful to Professor V. I. Ovcharenko for his useful comments. This work is supported by the Russian Foundation for Basic Research via RFFI-10-0296011, RFFI-13-02-00374, RFFI-10-03-00075, and RFFI-1002-00140, the Ministry of education and science of Russia (Grants 12.740.11.0026 and 8436), and by the Ural branch of Russian Academy of Science through the young-scientist program. All the calculation were performed on the "Uran" cluster of the IMM UB RAS. 
*streltsov@imp.uran.ru

${ }^{1}$ P. Gütlich and H. A. Goodwin, in Spin Crossover in Transition Metal Compounds I, II, and III, Topics in Current Chemistry (Springer, Berlin, 2004).

${ }^{2}$ N. A. Babushkina, A. N. Taldenkov, S. V. Streltsov, T. G. Kuzmova, A. A. Kamenev, A. R. Kaul, D. I. Khomskii, and K. I. Kugel, arXiv:1301.2241.

${ }^{3}$ Y. Ogawa, S. Koshihara, K. Koshino, T. Ogawa, C. Urano, and H. Takagi, Phys. Rev. Lett. 84, 3181 (2000).

${ }^{4}$ H. J. Shepherd, S. Bonnet, P. Guionneau, S. Bedoui, G. Garbarino, W. Nicolazzi, A. Bousseksou, and G. Molnár, Phys. Rev. B 84, 144107 (2011).

${ }^{5}$ S. V. Streltsov and N. A. Skorikov, Phys. Rev. B 83, 214407 (2011).

${ }^{6}$ A. Hauser, Top. Curr. Chem. 233, 49 (2004).

${ }^{7}$ S. Cobo, D. Ostrowskii, S. Bonhommeau, G. Molnár, L. Salmon, K. Tanaka, and A. Bousseksou, Top. Curr. Chem. 130, 9019 (2008). ${ }^{8}$ P. Gütlich, A. Hauser, and H. Spiering, Angew. Chem. Int. Ed. Engl. 33, 2024 (1994).

${ }^{9}$ J. F. Létard, P. Guionneau, and L. Goux-Capes, Top. Curr. Chem. 235, 221 (2004).

${ }^{10}$ V. I. Ovcharenko, S. V. Fokin, G. V. Romanenko, V. N. Ikorskii, E. V. Tretyakov, S. F. Vasilevsky, and R. Z. Sagdeev, Mol. Phys. 100, 1107 (2002).

${ }^{11}$ V. I. Ovcharenko, K. Maryunina, S. V. Fokin, E. V. Tretyakov, G. V. Romanenko, and V. N. Ikorskii, Russ. Chem. Bull. 53, 2406 (2004).

${ }^{12}$ E. V. Tretyakov, S. E. Tolstikov, A. O. Suvorova, A. V. Polushkin, G. V. Romanenko, A. S. Bogomyakov, S. L. Veber, M. V. Fedin, D. V. Stass, E. Reijerse et al., Inorg. Chem. 51, 9385 (2012).

${ }^{13}$ J. W. Abraham, K. Balzer, D. Hochstuhl, and M. Bonitz, Phys. Rev. B 86, 125112 (2012).
${ }^{14}$ A. Olivetti, J. Barré, B. Marcos, F. Bouchet, and R. Kaiser, Phys. Rev. Lett. 103, 224301 (2009).

${ }^{15}$ See Supplemental Material at http://link.aps.org/supplemental/ 10.1103/PhysRevB.87.024425 for the list of the atomic positions for $T=240 \mathrm{~K}$ and $T=110 \mathrm{~K}$.

${ }^{16}$ R. N. Musin, P. V. Schastnev, and S. A. Malinovskaya, Inorg. Chem. 31, 4118 (1992).

${ }^{17}$ V. Ovcharenko, G. V. Romanenko, K. Y. Maryunina, A. Bogomyakov, and E. V. Gorelik, Inorg. Chem. 47, 9537 (2008).

${ }^{18}$ E. M. Zueva, E. R. Ryabykh, and A. M. Kuznetsov, Russ. Chem. Bull. 58, 1654 (2010).

${ }^{19}$ S. Vancoillie, L. Rulísek, F. Neese, and K. Pierloot, J. Phys. Chem. A 113, 6149 (2009).

${ }^{20}$ M. V. Fedin, S. L. Veber, K. Y. Maryunina, G. V. Romanenko, E. A. Suturina, N. P. Gritsan, R. Z. Sagdeev, E. G. Ovcharenko, and V. I. Bagryanskaya, J. Am. Chem. Soc. 132, 13886 (2010).

${ }^{21}$ A. V. Postnikov, A. V. Galakhov, and S. Blügel, Phase Trans. 78, 689 (2005).

${ }^{22}$ P. Giannozzi, S. Baroni, N. Bonini, M. Calandra, R. Car, C. Cavazzoni, D. Ceresoli, G. L. Chiarotti, M. Cococcioni, I. Dabo et al., J. Phys.: Condens. Matter 21, 395502 (2009).

${ }^{23}$ J. P. Perdew, K. Burke, and M. Ernzerhof, Phys. Rev. Lett. 77, 3865 (1996).

${ }^{24}$ V. Anisimov, F. Aryasetiawan, and A. Lichtenstein, J. Phys.: Condens. Matter 9, 767 (1997).

${ }^{25}$ A. I. Liechtenstein, V. I. Anisimov, and J. Zaanen, Phys. Rev. B 52, 5467 (1995).

${ }^{26}$ S. V. Streltsov and D. I. Khomskii, Phys. Rev. B 86, 035109 (2012).

${ }^{27} \mathrm{~J}$. B. Goodenough, in Magnetism and the Chemical Bond (Interscience-Wiley, New York, 1963). 